\title{
Surface plasmon resonance sensor for detecting of arsenic in aqueous solution using polypyrrole-chitosan-cobalt ferrite nanoparticles composite layer
}

\begin{abstract}
The detection and measurement of low concentrations of arsenic (V) are the subjects of intense research interest in chemistry and environmental activity. In this research, a polypyrrole-chitosan/cobalt ferrite nanoparticles composite layer was prepared using an electrodeposition method on a gold-coated glass slide. The composite layer was characterized using field emission scanning electron microscopy, energy-dispersed spectroscopy, atomic force microscopy, and a high surface stylus profilometer. The composite layer was used to detect the arsenic in water, and the sensor limitation was about $0.001 \mathrm{ppm}$. The composite layer was tested using atomic-force microscopy before and after the detection of arsenic. As a result, the roughness was disoriented, as the arsenic was bound on the surface of the composite layer.
\end{abstract}

Keyword: Arsenic; Polypyrrole-chitosan; Cobalt ferrite nanoparticles; Surface plasmon resonance 